Service social

\title{
Le développement de la pensée critique, condition de l'avancement de la pratique sociale
}

\section{Madeleine Beaudry}

Volume 42, numéro 2, 1993

Recherche et pensée critique

URI : https://id.erudit.org/iderudit/706614ar

DOI : https://doi.org/10.7202/706614ar

Aller au sommaire du numéro

Éditeur(s)

École de service social de l'Université Laval

ISSN

1708-1734 (numérique)

Découvrir la revue

Citer ce document

Beaudry, M. (1993). Le développement de la pensée critique, condition de

l'avancement de la pratique sociale. Service social, 42(2), 3-6.

https://doi.org/10.7202/706614ar d'utilisation que vous pouvez consulter en ligne. 


\section{AVANT.PROPOS}

\section{Le développement de la pensée critique, condition de l'avancement de la pratique sociale}

Devant l'ampleur des problèmes sociaux actuels, tels que la pauvreté, le chômage, la violence, l'alcoolisme, l'abus de drogues, et les conséquences malheureuses qui en découlent tant pour les individus, les familles que pour les communautés, les travailleuses sociales et les travailleurs sociaux doivent prendre des décisions de plus en plus complexes. Quelles approches théoriques favoriser? Quelles méthodes de recherche ou d'intervention adopter pour résoudre ces problèmes? Quels programmes juger prioritaires? Qui fera quoi, pour qui, quand, garantissant quels effets pour résoudre quels types de problèmes particuliers? La prise de décision est une activité qui se situe au cœur même de la pratique du service social. Chaque jour et pour chacun de ses clients, I'intervenante ou l'intervenant social doit analyser la réalité à laquelle elle ou il fait face et porter un jugement qui oriente ses actes. Pour offrir le meilleur service possible à ses clientes et à ses clients, il est important qu'elle ou qu'il s'interroge sur ses certitudes, développe ses connaissances, précise ses stratégies, évalue les risques et vérifie les conséquences ou les résultats de son action. En somme, chacun doit développer sa pensée critique afin de démontrer de manière convaincante et appuyée la valeur de ce qu'il accomplit dans son intervention. "Critical thinking skills, knowledge, and attitudes can help clinicians to choose wisely among options - to select options that, compared to others, are most likely to help clients» (Gambrill, 1993).

Les articles qui font partie de ce numéro de la revue présentent différentes facettes de la pensée critique. Une de ces facettes a trait au développement des connaissances et à l'impératif besoin d'utiliser des méthodes de recherche 
adéquates. À cet égard, Michel Dorais expose de manière originale et avec une grande clarté différents types de recherche qualitative. Dans un tableau synthèse, il propose cinq aspects qui permettent de répertorier les différentes pratiques en ce domaine, puis il les développe et les illustre par des exemples de recherche. Celui ou celle qui veut découvrir ou approfondir les concepts et la réalité en constante évolution dans ce domaine lira avec grand intérêt cet article synthèse.

L'avancement de la pensée critique implique l'amélioration de ses capacités d'observation et d'analyse. L'article de Marie-Andrée Couillard et de Ginette Côté en est un bel exemple. À l'ère du partenariat et des rapports mouvants entre les groupes communautaires et l'État, Mmes Couillard et Côté observent et décrivent avec acuité les groupes de femmes de la région de Québec et leurs liens avec le réseau de la santé et des services sociaux. Suivant une méthode qualitative, elles dégagent des éléments qui colorent les conditions dans lesquelles le communautaire se réalise. Ainsi, elles soulèvent des paradoxes, mettent en lumière des effets non prévus et précisent certaines difficultés qui affectent ces rapports. Ce sont là des activités inhérentes à l'avancement de la pensée critique.

Savoir regrouper et réorganiser les résultats de recherche souvent contradictoires de façon à former un tout cohérent et compréhensible s'impose comme point de départ du raisonnement critique. Comment poser des questions pertinentes, comprendre les courants théoriques qui structurent notre pensée, interpréter des résultats sans d'abord comprendre le fondement des connaissances dans un domaine donné? Dans son article sur les dynamiques traumatisantes des abus sexuels et leurs conséquences à long terme, Dominique Damant illustre un point important de l'activité scientifique, la revue des écrits. En se basant sur un modèle théorique, elle regroupe les nombreuses conséquences reliées aux abus sexuels. Une bonne connaissance de cette problématique permettra aux intervenantes de prendre des décisions plus éclairées en ce qui a trait aux besoins des clientes qui ont malheureusement vécu de telles expériences traumatisantes.

Établir le plus clairement possible les critères sur lesquels se fonde le jugement constitue un autre point important de la pensée critique. Par exemple, dans le contexte de la protection de la jeunesse, quelle est l'information pertinente pour statuer sur la compromission de la sécurité ou du développement de l'enfant? Existe-t-il un seuil critique à partir duquel l'intervention serait nécessaire? Ce sont là les questions auxquelles Aline Vézina et Daniel Pelletier ont voulu répondre afin d'offrir aux praticiens un moyen concret, un instrument de mesure, pour nuancer et objectiver leur jugement.

Porter un jugement critique sur sa pratique implique aussi d'examiner le contexte ou les conditions dans lesquelles s'exerce cette pratique (Laforest et Redjeb, 1991 ; Gambrill, 1990). À I'heure de la rareté des emplois, de la pression morale accrue pour augmenter la charge de travail et de l'attitude souvent blâmante envers les personnes qui ne peuvent supporter cet environ- 
nement de travail, il importe de se pencher sur les relations entre les variables organisationnelles et la capacité des praticiens de prendre des décisions éclairées. Sur ce point, l'étude de Jean-Pierre Villeneuve et d'André Beaudoin fournit une information fort pertinente en précisant comment le cadre de travail est associé à l'épuisement professionnel des intervenants sociaux.

C'est un signe d'intelligence et non d'incompétence que de se poser des questions sur son intervention. II peut y avoir plusieurs types de questions. Par exemple: Qu'est-ce qui a facilité le changement chez ce client? Qu'est-ce que j'ai fait comme intervenant ou comme intervenante qui a permis ce changement? Quelle évidence me permet de dire qu'il y a un lien entre ce que j'ai fait et le changement qui s'est produit? L'évaluation sommative et formative de ses interventions permet de découvrir de nouvelles façons de maximiser l'efficacité de la pratique sociale. Dans leur étude longitudinale descriptive à caractère exploratoire, Georgette Béliveau, Martin Poulin et Ginette Beaudoin dégagent les impacts de l'informatisation des données de service social en milieu hospitalier. Ils permettent ainsi d'évaluer les changements qu'entraîne une telle innovation sur le plan de l'accomplissement de la tâche, de la pratique professionnelle, des communications, de la confidentialité, du contrôle et de l'évaluation, de l'image professionnelle des intervenants, de même que des craintes et des attentes. Dans un autre article portant sur l'analyse $d^{\prime}$ 'un programme d'éducation sexuelle administré auprès de garçons en difficulté d'adaptation, France Langevin et Jocelyn Lindsay apportent une contribution originale dans un domaine d'intervention relativement peu exploré en service social.

D'une manière différente, mais tout aussi importante pour le développement de l'enseignement ou de la pratique, décrire précisément ses expériences d'enseignement ou d'intervention et les analyser facilite la répétition du processus par les personnes intéressées, tout en évitant la reproduction des erreurs. Dans son article sur la supervision pédagogique dans l'approche de conscientisation, Judith Breton retrace avec rigueur les différentes phases d'une expérience éducative en processus de formation scolaire en techniques de travail social. L'analyse de cette expérience originale et novatrice ouvre sur des perspectives de développement pour cette approche de la supervision. Dans la note de recherche, Pascaline Cosse éclaire le lecteur sur le processus d'implantation d'un mode d'intervention sociale de plus en plus visible, soit le développement économique communautaire. L'évaluation d'une intervention de groupe portant sur l'émancipation des femmes par la création $d^{\prime}$ 'une entreprise collective soulève plusieurs questions sur les conditions nécessaires à la réussite de telles expériences novatrices. Du même coup, ces derniers articles illustrent les avantages liés à l'évaluation.

Que la lecture de ce numéro de la revue Service social vous permette d'exercer votre pensée critique! 


\section{Références bibliographiques}

GambriLl, E. (1993). "What Critical Thinking Offers to Clinicians and Clients», The Behavior Therapist, vol. 16, $\mathrm{n}^{\circ} 6: 141-146$.

GambrilL, E. (1990). Critical Thinking in Clinical Practice. San Francisco: Jossey-Bass Inc.

LAforest, M. et B. Redjeb (1991). «Le service social et les conditions de sa pratique: un rapport à examiner », Service social, vol. 40, n $2: 89-103$. 